\title{
The 1.3-meter Robotically Controlled Telescope: Developing a Fully Autonomous Observatory
}

\author{
Richard Gelderman \\ Western Kentucky University, Dept. of Physics and Astronomy, \\ Bowling Green, Kentucky, USA 42101
}

\begin{abstract}
The former KPNO 1.3-meter telescope is being refurbished and automated by a consortium of U.S. institutions, headed by Western Kentucky University, with the goal of a 2002 recommissioning as the Robotically Controlled Telescope (RCT). The 1.3-meter RCT will operate in fully autonomous mode to obtain guided images for a variety of research and education programs. Distinctions between a fully autonomous versus robotic observatory are presented, along with a discussion of why fully autonomous operation is necessary for increased productivity of small telescopes.
\end{abstract}

\section{The RCT - Honoring the Past}

In 1964, with NASA funding, a new 1.3-meter tèlescope was commissioned on Kitt Peak, in Arizona, and named the "Remotely Controlled Telescope." As the name implies, the RCT was designed to be operated remotely with no people actually at the telescope. The project was originally headed by the Kitt Peak National Observatory (KPNO) Space Sciences Division, intended to develop techniques for operating space-based telescopes. This effort included state-of-the-art (for the mid 1960's) motion encoders, a 'powerful' computing system (so compact that it fit inside a single large room), and a communication network based on phone line connections from the mountain to the KPNO headquarters roughly $75 \mathrm{~km}$ away in Tucson. However the poor results from engineering tests performed at the downtown headquarters before the telescope was installed on the mountain prompted KPNO to reevaluate its goals.

In response to the Whitford Committee (1964) report, the purpose of the 1.3-meter's automation was shifted to an attempt to enhance the productivity of small telescopes through remote operation. In 1969, control of the telescope was shifted to KPNO's Stellar Division and the attempts at remote operation were directed toward demonstrating that a ground-based observatory could be operated from a distance of many miles. Eventually, this experiment was also not deemed to be successful and the KPNO 1.3-meter telescope was refurbished for classical, on-site operations. Shortly thereafter, the troublesome aluminum mirror was replaced with a honeycombed lightweight Cer-Vit primary. For the next 25 years the 1.3-meter was a productive small telescope, first with a photoelectric photometer and later as a testbed for pace-setting infrared instrumenta- 
tion. Finally, in 1995, due to pressure from a decreasing budget, the 1.3-meter was closed by KPNO.

In 1999, the National Optical Astronomical Observatory announced an opportunity to "assume responsibility for operation of the Kitt Peak 1.3-meter telescope." A collaboration of US astronomers, headed by Western Kentucky University, successfully proposed to refurbish the 1.3-meter and automate the observatory for operation as the "Robotically Controlled Telescope." The RCT Consortium was established to manage the refurbishment and establish scientifically and educationally productive operations. Funding has been provided by contributions from the RCT Consortium partner institutions and a generous award from the NASA Office of Space Science. The contract to refurbish, automate, and provide maintenance for the new RCT was awarded in the spring of 2000 to EOS Technologies, Inc. of Tucson, Arizona.

\section{Defining a Fully Autonomous (vs. Robotic) Observatory}

In general, a robot is a mechanism guided by automatic controls but is not necessarily a mechanism which operates fully autonomously. While the ultimate robot might be fully autonomous (or even self-aware, as in Karel Capek's 1920 science fiction play Rossum's Universal Robots, which first introduced the term "robot") the term is commonly used for any device which performs a repetitive task without constant human intervention. In astronomy, there are many robotic observatories but very few fully autonomous ones.

With a hope that our discussions will benefit from a common vocabulary we offer the following definitions for stages of robotic observing.

Classical On-site observing involves having all observers and operators at the site, with the observatory completely under local control.

Remote observing allows the observer to have some level of instrument control from a distant location but still requires a local telescope operator at the site. Many facilities routinely offer remote observing (e.g., the ARC 3.5-meter at Apache Point, New Mexico and most of the large telescopes on Mauna Kea).

Unmanned observing requires enough computer control that remote observers/ operators can control all aspects of the observatory functions. Verification and tasks such as acquiring guide stars are performed manually. Many ground based observatories and some early orbiting observatories (e.g., the International UItraviolet Explorer) operated in unmanned mode, without being robotic.

Robotic observing requires automatic control of the instruments but involves pre-scheduled observations submitted as a queue and the need for pre-selected guide stars. Modern space-based observatories (e.g., the Hubble Space Telescope) and a number of small ground based telescopes (e.g., the Fairborn Observatory or the Katzmann Automated Imaging Telescope) are productive robotic telescopes.

Fully Autonomous observations involve the ability to constantly rearrange observing schedules to optimize performance, much as a well-trained human astronomer would adjust to changing sky conditions. 
The refurbishment of the 1.3-meter will increase its capabilities to allow for fully autonomous operation, surpassing both "remote" and "robotic" modes of operation. We chose, however, to avoid the name "Fully Autonomous Telescope" in favor of the more attention grabbing term "robotic" and the chance to honor the past by reclaiming the RCT acronym. Despite our misuse of the terms, we argue that robotic and fully autonomous are not synonymous and that that fully autonomous is a significantly more stringent definition than is robotic.

The key to fully autonomous observing is software which acts as a scheduler, allowing for flexibility in response to changing conditions and complex science requirements. A robotic telescope, with each night's observing scripted in advance, will be productive with a well defined and repetitive program. An optimized, fully autonomous scheduler, however, will allow a diverse collection of targets to be observed without having to specify their exact place in the program's sequence. It is not hard to imagine situations where a robotic observatory will lose productivity whenever the observing program is no longer well defined and repetitive or for some other reason an efficient schedule cannot be predetermined. For instance, robotic telescopes are rarely flexible enough to overcome the mechanical and/or weather problems which challenge any observing program; and generally can not deal with the desirable complexities of observations coordinated among multiple telescopes.

\section{Global Networks of Fully Autonomous Telescopes}

One interpretation of Small Telescope Astronomy on Global Scales is the establishment of global networks of small aperture telescopes. Coordinating such a network imposes all the demands of operating a single observatory, while adding concerns related to distance, non-standard hardware, and the needs of a diverse collection of users. To be successful, a global network must allow for realistically complex observing programs while keeping the costs low enough for long term and large scale projects to be feasible. New observers should not have to immerse themselves in specialized procedures in order to make use of the network. For instance, an observer should be able to schedule a single observation to run each night for weeks, or years, without devoting weeks, or years, of her/his life to monitoring the progress of the observing.

A global network of small telescopes will be most efficient and effective if the telescopes can operate in fully autonomous mode. A major hurdle to establishing such a network is the personnel costs of coordinating a full time network with on-site operators. Examples of successful global networks tend to be either wellfunded efforts (at least compared to the typical small telescope initiative; e.g., the Global Oscillation Network Group or the Very Long Baseline Interferometry (VLBI) radio network) or low- to non-funded collaborations coordinated on a part-time basis (e.g., the Whole Earth Telescope). However, many astronomers have a full-time load of responsibilities beyond research and are unable to commit to an additional load of observing multiple times each week. The availability of eager and capable graduate student researchers can solve the personnel dilemma for some observatories. But as a general rule, hiring and retaining a group of top notch telescope operators can be prohibitively expensive. Thus a global network should at minimum be based upon robotic observatories and only a 
fully autonomous system can hope to come close to replacing the adaptability of human observers.

\section{Refurbishing and Automating the RCT}

The 1.3-meter RCT is being refurbished and automated as one piece of the STARBASE global network of telescopes. The RCT is a Cassegrain telescope built by Boller \& Chivens according to some interesting specifications, many of which presage the design of modern observatories such as WIYN, Keck, or Gemini. The mount and dome for the RCT are relatively undersized. Its german equatorial mount was designed for a 0.9-meter telescope with an open truss support structure designed for its lightweight (originally spun aluminum) 1.3meter primary mirror. The dome also is typical of a 0.9 -meter observatory, since the intended remote operations could be accomplished with less extra space than human observers might require.

After an engineering assessment, the RCT Consortium chose to keep the existing $f / 13.5$ optics, the original mount, and the 36 year old worm gear drives. We also chose to keep the telescope in its original dome at the existing site, despite its proximity to the KPNO administration building and asphalt parking lots. Instead, we have focussed our efforts on refurbishing the encoders and computer interfaces to provide input to custom software for fully autonomous observing.

Observatory Control Computer: The central nervous system of the RCT is the Observatory Control computer, which accepts input, makes decisions, and outputs commands and messages as it coordinates and controls the activities within the entire observatory. For instance, based on data provided by the weather station and sky monitors, the Observatory Control computer makes the determination to open or close the dome, and then issues commands to the dome slit controller and updates the status in the event log.

The Observatory Control computer incorporates the Scheduler program, the event $\log$, and searchable star catalogs; as well as interfacing through the local area network (LAN) to all other observatory functions. Self-contained instruments (e.g., the meteorological station, $15 \mathrm{MHz}$ clock, and uninterruptible power supply) interface directly to the Observatory Control computer. Other instruments, such as the CCD camera, the autoguider, or the controller for the telescope systems, interface with the Observatory Control through their own separate computers.

Scheduler: Fully autonomous operation is derived from the function of the Scheduler. The Scheduler accepts observation requests from users and, during operation, determines the next observation based on various constraints.

Requests consist of 1) details about the user's identity and the target, 2) scheduling parameters (e.g., range of dates to complete observations, worst acceptable seeing conditions, maximum airmass, maximum moon phase, \& minimum separation from the moon), and 3) observation requirements (e.g., integration time, filter, \& calibration needs). A Scheduler unit consists of a single observation of a target. The ability to request an observing sequence of multiple images of the same target (e.g., with varying integration times, through different filters, or as identical observations) is easily accomplished with the request edi- 
tor; however, once submitted, such a request is treated as multiple observations by the Scheduler.

The operation of the Scheduler is tuned by adjustment of weighting parameters which relate to various observing decisions such as quality, quantity, timeliness, and fairness. The quality weighting factor compares the scheduling parameters submitted by the user to the current conditions. Potential observations may be excluded if the conditions do not meet the requirements or may be given a lower weight depending on how far the current conditions are from the requested optimum conditions. The quantity weighting factor controls the emphasis that the Scheduler places on maximizing the total number of observations completed within a given time period. Potential observations can be given a higher weight if they have shorter integration times or require a minimal slew time from the previous position. The timeliness weighting factor allows the Scheduler to prefer observations which will not be possible to obtain at a later date. The fairness weighting factor ensures that over a given accounting period the allocation of time within the Consortium is distributed according to either a fixed number of hours or predetermined percentages. The observing request which is returned to the telescope for execution is the one with the maximum total for all of the weighting factors. If the telescope is unable to complete the observation for any reason then the request is returned to the Scheduler for reconsideration.

Telescope Control Computer: The Telescope Control computer manages the operation of the telescope drive motion and limits, focus, temperature sensors, filter wheels, calibration lights, observatory security lights, mirror covers, dome shutter, and dome azimuth motion. The various components of the telescope system interface to the Telescope Control computer through new digital I/O gate arrays. The existing DC servo drive motors and worm gears are monitored and controlled with new PMAC motion control cards, switch mode drive amplifiers, and on-axis incremental encoders. Axis position and drive motor velocity are used as feedback to establish a stable and accurate servo loop. Multiple levels of limit protection are provided by the software and hardware. These range from a warning to human operators who might issue a command to move beyond the predefined range of operation, through a software commanded reduction in speed as a limit is approached, to an interruption of power to prevent catastrophic failures. The Telescope Control computer also controls the possibility of german flips and establish zenith and stow positions for the scope. The focus can be monitored and either adjusted remotely or as a part of fully autonomous operations. The secondary mirror position is controlled automatically to compensate for expansion of the telescope's open truss structure using input from the various temperature sensors. There are 16 available slots for broadband and narrowband filters, distributed between two filter wheels. The brightness level of flat field calibration lamps and security lights within the observatory are adjustable through a computer interface. The dome shutter and mirror covers can open and close the observatory automatically in threatening conditions; such as high wind, threat of precipitation, or imminent power failure. The dome can be moved independently of the telescope, as required during flat-field calibration. However, for normal operation, as the telescope slews or tracks across the sky, the dome azimuth is monitored and controlled to keep 
the RCT's relatively undersized dome slit aligned with the telescope. A virtual control panel allows remote users to easily interface with the Telescope Control computer, either taking control of the observatory systems or simply monitoring its robotic operation.

Autoguider: The RCT's off-axis autoguider employs a pick-off mirror, driven by an X-Y stage to the position selected within the available field of view, to feed the image to the guide camera. Suitable guide stars are selected by the Observatory Control computer from its searchable star catalogs. The autoguider computer moves the autoguider's X-Y stage to the expected position for the selected guide star and executes a search pattern to center the guide probe on the selected star. It is possible to focus the telescope from the image of the guide star, with the seeing (measured from the size of the guide star's point spread function) returned to the Observatory Control computer for use by the Scheduler. When enabled, the autoguider computer controls and monitors the position of the probe while the output from the autoguider program provides the guider offsets to the Telescope Control computer.

Camera: The RCT is a single instrument telescope. We have an imager with a thinned, back illuminated SITe $2048 \times 2048$ pixel CCD. It is operated through its own computer and linked to the RCT Observatory Control computer.

\section{Research and Educational Goals for the RCT}

The RCT Consortium is organized around common educational goals and common research projects. Our overall goal is to provide high quality astrophysical images for use in these education and research programs. The four main research areas are: broadband photometry of moderately dense star fields to identify extrasolar planets (Everett \& Howell 2001); broadband photometry of quasars and blazars (Clements \& Carini 2001); narrowband imaging of Galactic and extragalactic nebulae (Buckalew et al. 2000); and rapid response to transient events such as gamma ray bursts.

Our educational program addresses the need for hands-on, inquiry-based learning. All of the projects will require students to work through the entire scientific process - from learning to frame productive questions, to performing good observations or experiments, to presenting to an audience the results of the project. Teacher-student teams will either participate in one of our main research programs or will be able to establish their own investigation with help from a mentor.

\section{References}

Buckalew, B., Dufour, R.J., Shopbell, P., and Walter, D.K. 2000, AJ, 120, 2402 Clements, S.D. and Carini, M.T. 2001, AJ, 121, 90

Everett, M.E. and Howell, S.B. 2001, PASP, submitted

Whitford et al. 1964, Ground-Based Astronomy: a Ten-Year Program, (Washington: National Academy of Sciences), Pub. No. 1234 